\title{
Pesquisa Científica da Área de Marketing: uma Revisão Histórica
}

\author{
Cláudio Hoffmann Sampaio \\ Marcelo Gattermann Perin
}

Este artigo apresenta um levantamento das características metodológicas básicas da produção científica de pesquisas empíricas publicadas no I EMA e nos últimos 16 ENANPADs, dentro da área de Marketing, visando avaliar as suas tendências, bem como comparar a sua evolução através dos anos. Dentre as variáveis analisadas para cada artigo estão: natureza da pesquisa; embasamento conceitual e questão de pesquisa; desenho de pesquisa; instrumento de medida e coleta de dados e apresentação dos resultados. De 527 artigos identificados, 407 respeitaram a abordagem positivista, 108 apresentaram cunho conceitual e 12 se enquadraram na abordagem fenomenológica ou interpretativista. A partir desta análise e do cruzamento das variáveis, apresenta-se a discussão e as conclusões da qualidade do conjunto de artigos avaliados.

\section{INTRODUÇÃO}

Nos últimos anos, alguns artigos publicados nos anais dos Encontros Anuais da Associação Nacional dos Programas de Pesquisa e Pós-Graduação (ENANPAD) e do I Encontro de Marketing da ANPAD (EMA), bem como nos principais periódicos científicos da área, procuraram trazer à tona a discussão sobre a pesquisa e produção científica da comunidade acadêmica brasileira, especificamente na área de Administração (Caldas, Tinoco, \& Chu, 2003; Cardoso, Pereira, \& Guerreiro, 2004; Gonçalves, Gonçalves, \& Augusto, 2004; Iizuka \& Sano, 2004; Tonelli, Caldas, Lacombe, \& Tinoco, 2004). Além dos artigos, alguns painéis e mesas redondas vêm analisando desde a qualidade até a continuidade dos trabalhos realizados e publicados em âmbito nacional dentro da Ciência da Administração. 
Este fato manifesta preocupação constante dos acadêmicos brasileiros, particularmente da área de Administração, no sentido de reavaliar a sua postura e procedimentos em relação à sua própria pesquisa acadêmica, bem como revisitar a produção nacional do conhecimento em Administração. Dentro desse contexto, a área específica de Marketing também vem buscando uma autoanálise consistente no que tange à sua tarefa de geração de conhecimento. Nesta linha, os acadêmicos da área de Marketing no Brasil têm demonstrado grande esforço no sentido de discutir a sua identidade e também os rumos desta disciplina no contexto nacional, especialmente a partir do final da década de 90, no escopo dos seus congressos acadêmicos de expressão na área de Marketing, ENANPAD e EMA. Essa atitude passa pela condução de vários estudos de diagnóstico da produção científica nacional (Botelho \& Macera, 2001; Campomar, 2005; Farias, 2004; Kovacs, Leão, Vieira, Barbosa, \& Dias, 2004; Perin, Sampaio, Froemming, \& Luce, 2000; Proença, Lopes, \& Meucci, 2005; Révillion, 2001; Rocha \& Barros, 2004; Vieira, 1998, 1999, 2000); pela avaliação de posturas metodológicas alternativas (Barros, 2002; Botelho \& Macera, 2001; Casotti, 1998; Cerchiaro, Sauerbronn, \& Ayrosa, 2004; Jaime Jr., 2000; Neves \& Giglio, 2004; Rocha \& Barros, 2004; Rocha, Barros, \& Pereira, 2005; Vilas Boas, Sette, \& Abreu, 2004); e pela proposição de caminhos de consolidação da disciplina no país (Campomar, 2005; Cardoso \& Faria, 2004; Casotti, 1999; D‘Angelo, 2003; Faria, 2004; Farias, 2004; Rocha et al., 1999; Rossi \& Hor-Meyll, 2001; Rossi \& Silveira, 1999; Vieira, Dias, Filho, \& Neto, 2002).

Buscando integrar-se nesse esforço, este artigo tem como objetivo apresentar um levantamento e discussão dos trabalhos publicados entre 1990 e 2005 nos anais do I EMA e dos ENANPADs, dentro da área de Marketing. Para tanto foram analisadas as estratégias e as metodologias utilizadas, visando avaliar as suas tendências, bem como comparar a evolução através dos anos.

\section{Método}

O procedimento metodológico utilizado foi o de desk research, envolvendo o conjunto de artigos científicos da área de marketing, publicados nos anais do I EMA e dos ENANPADs, no período de 1990 a 2005. De fato, este estudo se valeu da base de dados gerada em levantamento anterior,que envolve as pesquisas científicas da década de noventa (Perin et al., 2000). Tal base foi complementada com novo levantamento, agora para o período de 2000 a 2005. 
O processo de identificação e seleção de artigos, incluindo-se os dados do primeiro levantamento, resultou em uma relação de 527 artigos para a análise. Foram considerados todos os artigos publicados na área de Marketing dos últimos 16 ENANPADs e do I EMA.

Dos 527 artigos identificados, 108 apresentavam cunho conceitual, 12 tinham abordagem fenomenológica ou interpretativista e 407 eram baseados em pesquisas empíricas que apresentaram método de pesquisa, envolvendo, direta ou indiretamente, alguma coleta de dados, com postura positivista. $\mathrm{O}$ estudo aqui Rossi e Silveira (1999) descrito envolve a avaliação metodológica dos artigos classificados como de base empírica, uma vez que as características metodológicas analisadas não se apresentam em artigos conceituais e não estão propriamente adaptadas para à avaliação de artigos de abordagem fenomenológica.

O processo de análise dos 407 artigos de pesquisas empíricas constituiu-se da verificação dos elementos metodológicos básicos de investigações científicas. As variáveis avaliadas para cada artigo selecionado, específicas para cada método de pesquisa (survey, qualitativa e experimental), foram adaptadas de Hoppen, Lapointe e Moreau (1997), seguindo procedimentos também adotados em Perin et al. (2000) e Froemming et al. (2000a, 2000b). No presente artigo, contudo, são relatados apenas os resultados das variáveis comuns aos três métodos de pesquisa considerados. Foram elas: natureza da pesquisa, justificativa, teorias de base, objetivo e questão de pesquisa, hipóteses ou pressupostos de base, modelo de pesquisa, mix de métodos, operacionalização das variáveis, descrição dos procedimentos metodológicos, tipo de dados, validade externa, validade nomológica, limites do estudo, recomendações para pesquisas e recomendações aplicadas.

A avaliação dessas variáveis foi realizada por dois pesquisadores de marketing; cada artigo foi analisado por apenas um avaliador. Contudo, buscando-se maior validade para o estudo, os critérios de análise foram amplamente discutidos entre os dois avaliadores e uma análise conjunta dos 15 primeiros artigos foi realizada. Além disto, os casos de artigos com aspectos duvidosos foram intercambiados entre os avaliadores.

A título de comparação dos resultados, o mesmo procedimento de análise foi aplicado aos 60 artigos publicados nos anais do I EMA de 2004. Neste caso, diagnosticou-se um total de 49 artigos de base empírica positivista.

Os dados, coletados por meio de formulário padronizado (Apêndice A), foram tabulados e processados por meio dos SPSS ${ }^{\circledR}$ e Excel ${ }^{\circledR}$. Os resultados da pesquisa são descritos a seguir. 


\section{Resultados das AnÁlises}

Os 527 artigos avaliados foram inicialmente classificados por postura epistemológica. Dentro da linha positivista, ademais, foram divididos por método de pesquisa, quais sejam, survey, qualitativa ou experimental (Hoppen et al., 1997). Foram também identificados os artigos de cunho conceitural (Figura 1).

\section{Figura 1: Frequiência de Artigos por Tipo de Pesquisa}

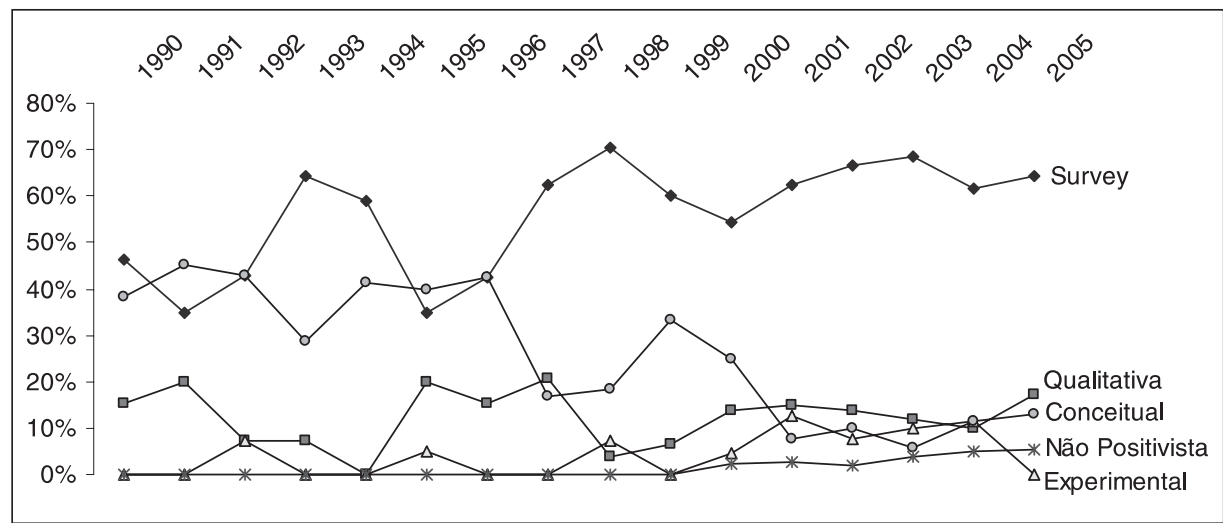

Nota: total de 527 artigos considerados.

Fonte: coleta de dados.

Dentro de uma linha positivista, a classificação do método de pesquisa como survey considerou elementos básicos, como o fornecimento de descrições quantitativas da população estudada, a realização de coleta de dados por meio de instrumentos estruturados e o uso de informações coletadas numa amostra da população analisada. Já as pesquisas definidas como qualitativas foram aquelas que objetivaram uma interpretação mais ampla do fenômeno social em estudo, visando à compreensão do estado da arte de situações, em que a prática se antecipa à teoria. Em geral, a técnica utilizada neste tipo de pesquisa foi a de estudo de casos. Por fim, os trabalhos considerados do tipo experimental foram aqueles que apresentaram manipulação deliberada de uma ou mais variáveis independentes para analisar as consequiências desta manipulação em uma ou mais variáveis dependentes, em ambiente controlado pelo investigador.

Observa-se, em relação aos métodos de pesquisa adotados, a predominância e o fortalecimento das pesquisas do tipo survey ao longo dos anos. Destaca-se, também, o avanço do uso da metodologia experimental nas pesquisas de marketing, a partir do ano 2000, o surgimento de artigos com a aplicação de metodologias de 
abordagem não positivista (2,3\%), com crescimento constante até o ano de 2005 $(5,3 \%)$. É de se destacar, ainda, a queda gradativa do percentual de artigos conceituais aprovados nos ENANPADs. Também é notória a dificuldade de afirmação dos estudos qualitativos e experimentais, não conseguindo ultrapassar as barreiras de $20 \%$ e $12 \%$, respectivamente.

A seguir são descritos os principais resultados relativos às variáveis analisadas, considerando tão somente os 407 artigos de base empírica identificados na área de Marketing.

\section{Natureza da Pesquisa}

A natureza da pesquisa foi classificada em exploratória, descritiva ou causal (Churchill, 1999), tendo sido limitada aos artigos identificados como de base empírica e postura epistemológica positivista.

No caso de uma pesquisa exploratória, trata-se de aprofundar conceitos preliminares, muitas vezes inéditos. Seu objetivo básico é desenvolver hipóteses e proposições que irão redundar em pesquisas complementares. Por outro lado, uma pesquisa descritiva tem como objetivo informar o pesquisador sobre situações, fatos, opiniões ou comportamentos da população analisada, buscando mapear a distribuição de um fenômeno na população estudada. Finalmente, a pesquisa causal foi considerada aquela que testa uma teoria e suas relações causais. A Figura 2 descreve a natureza das pesquisas em marketing publicadas nos periódicos analisados.

Figura 2: Frequiência de Artigos por Natureza de Pesquisa

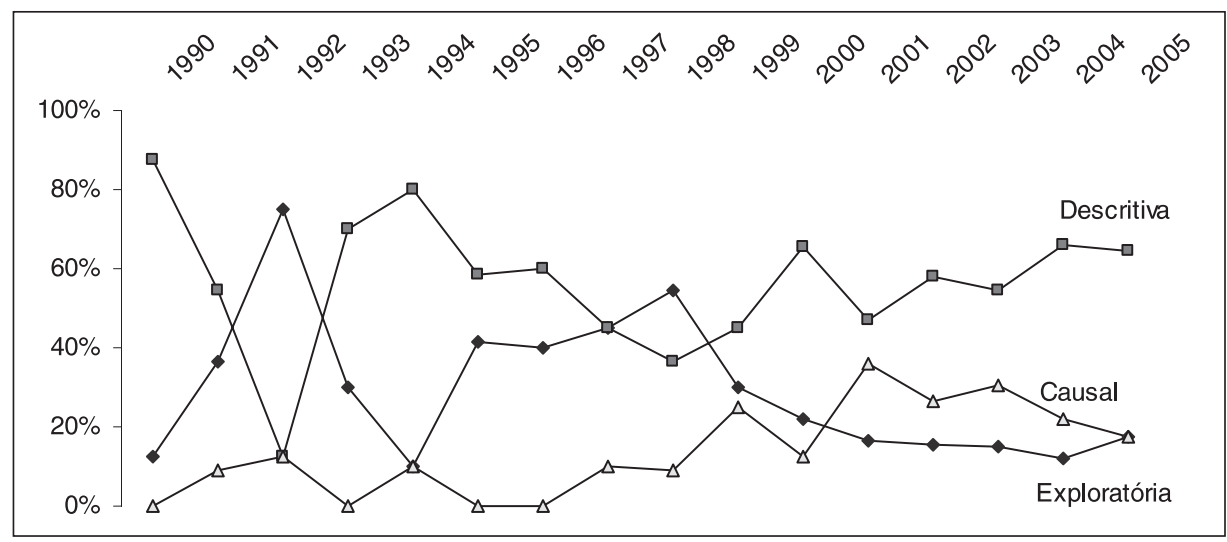

Nota: total de 407 artigos considerados como de base empírica e abordagem positivista.

Fonte: coleta de dados. 
A alteração mais evidente na natureza de pesquisa, já a partir de 1998, fica por conta da inversão de enfoque das pesquisas entre o caráter exploratório e o causal, tendo o segundo ultrapassado o primeiro no interesse dos pesquisadores da área de Marketing, mesmo que momentaneamente, no período de 2001 a 2004. Os artigos de natureza descritiva seguiram o seu domínio ao longo do tempo, ampliando a sua distância para as duas outras naturezas de pesquisa.

Por outro lado, nota-se uma estabilização das publicações de natureza exploratória a partir de 2000, após um comportamento errático na década de 90 , demonstrando a redução proporcional com a preocupação em aprofundar conceitos preliminares ou melhor definir novos conceitos da área.

\section{Apresentação e Relevância do Problema de Pesquisa}

Esta categoria trata de dimensões que identificam a fundamentação conceitual utilizada e a relevância do estudo para a área, bem como os objetivos da pesquisa apresentados e suas hipóteses. A Figura 3 apresenta os resultados da análise quanto a este item, apresentado os gráficos de freqüência das referidas dimensões.

Em relação à justificativa, registrou-se apenas se o artigo analisado apresentava justificativa ou relato de importância da pesquisa. A teoria de base foi considerada aprofundada, quando o artigo continha uma descrição detalhada do fenômeno em estudo do ponto de vista dos principais autores da área ou de uma linha de estudo específica de um grupo de autores. Caso a descrição do fenômeno apresentasse pouco detalhamento, mas ainda dentro do fenômeno em estudo, considerou-se a teoria de base como superficial. Em qualquer outro caso, considerou-se a teoria como ausente ou não relacionada.

Quanto ao objetivo e à questão de pesquisa, avaliou-se se o artigo descrevia ou não o problema de pesquisa e/ou objetivo geral. O problema de pesquisa implica uma questão que envolve dificuldade teórica ou prática, cuja resposta ou solução se quer encontrar. Da mesma forma, as hipóteses ou pressupostos de base foram apenas considerados como existentes (declarados) ou não para cada artigo analisado.

Conforme a Figura 3, os artigos apresentaram, em termos gerais, uma preocupação com a apresentação e a relevância do problema de pesquisa. Grande parte dos artigos apresentou justificativa do estudo, bem como objetivos e questão de pesquisa. A quase totalidade dos artigos apresentou a fundamentação teórica para a pesquisa descrita, entre aprofundada e superficial. 
No entanto uma análise mais aprofundada a respeito do comportamento dessas variáveis ao longo dos anos permite a constatação de alterações significativas em relação a duas das dimensões da apresentação e relevância do problema de pesquisa. Quanto ao uso de teorias de base, percebe-se que os autores procuraram aprofundar a fundamentação teórica de seus artigos, havendo-se registrado diminuição de artigos com teorias de base ausentes. Este fato talvez tenha justificativa no próprio aumento de pesquisas do tipo experimento e de natureza causal que, por si só, exigem maior aprofundamento teórico anterior para a sua operacionalização. Também é de se registrar a constância na atenção com a especificação do objetivo e questão de pesquisa, além da dimensão de justificativa.

\section{Figura 3: Frequiência de Artigos por Apresentação e Relevância do Problema de Pesquisa}
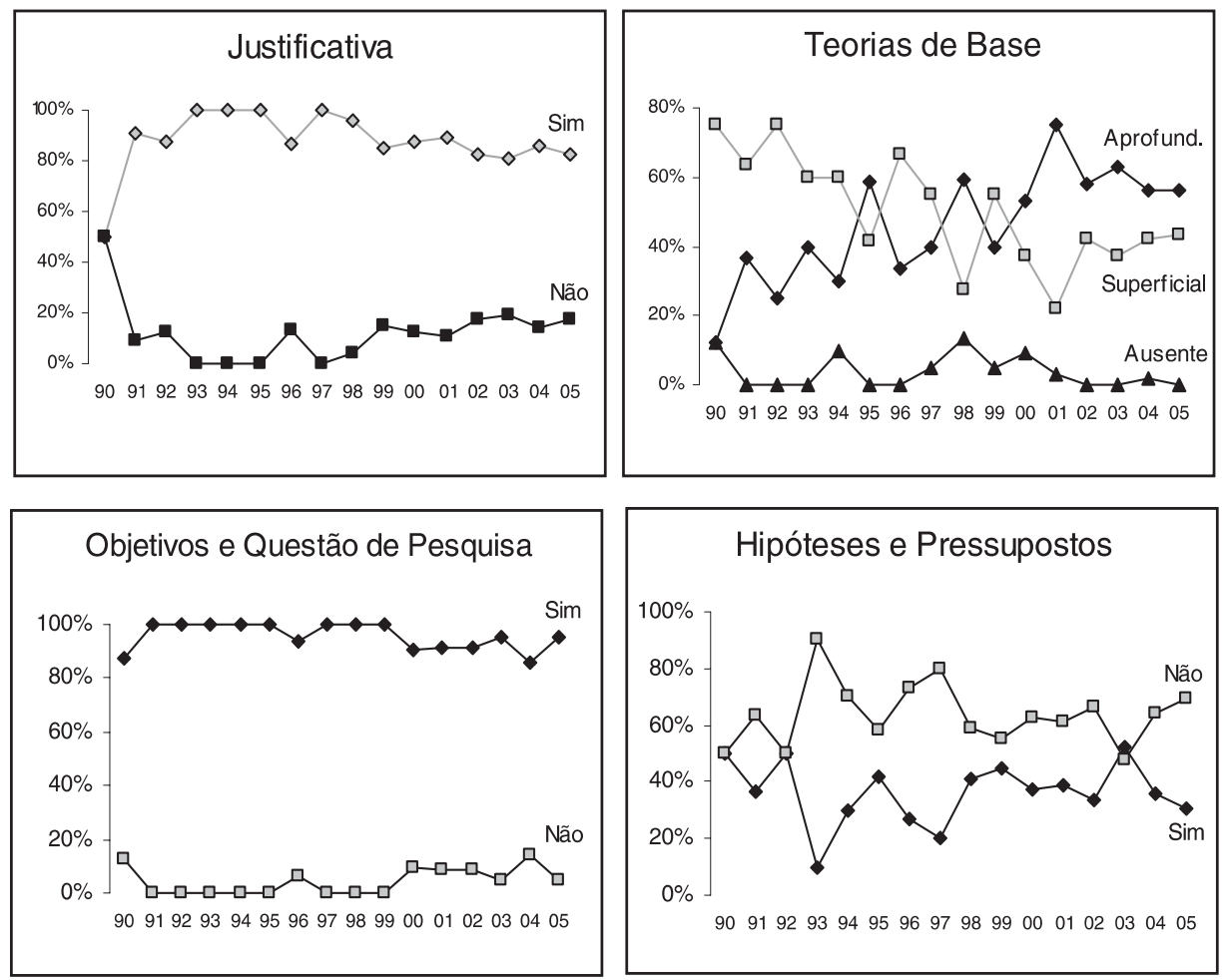

Nota: total de 407 artigos considerados como de base empírica e abordagem positivista.

Fonte: coleta de dados. 


\section{Desenho de Pesquisa}

Esta categoria engloba as dimensões relacionadas com o modelo de pesquisa, utilização de mix de métodos, operacionalização das variáveis do estudo e descrição dos procedimentos metodológicos utilizados. A presença dessas dimensões foi verificada em cada um dos artigos analisados. A Figura 4 resume os resultados obtidos.

\section{Figura 4: Freqüência de Artigos por Desenho de Pesquisa}
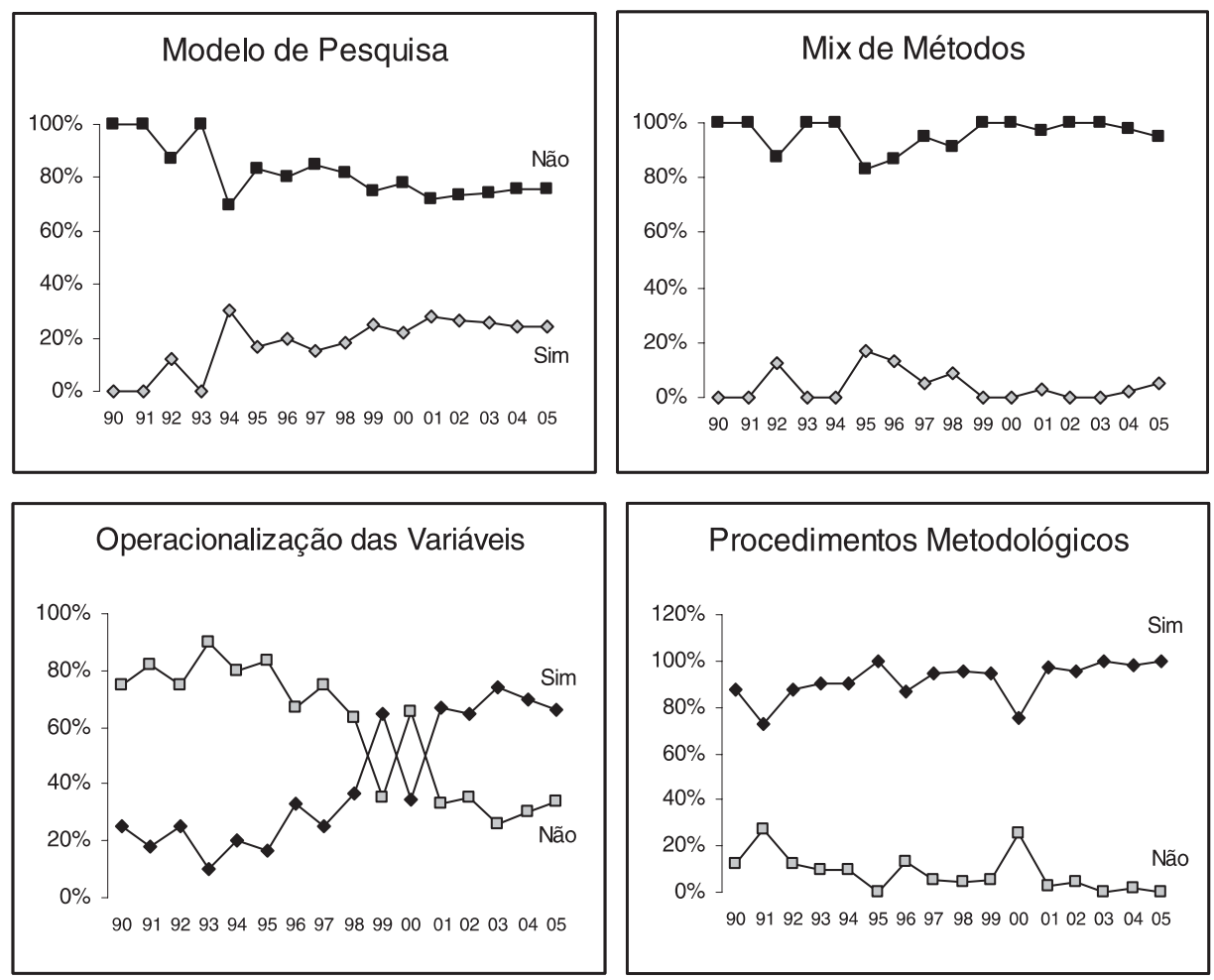

Nota: total de 407 artigos considerados como de base empírica e abordagem positivista. Fonte: coleta de dados.

Como modelo de pesquisa considerou-se o posicionamento do objeto em estudo dentro da base teórica ou de uma descrição mais ampla do fenômeno estudado, podendo ser gráfico ou não. Quanto ao mix de métodos levou-se em conta a utilização de mais de um método de pesquisa para avaliar a mesma questão de pesquisa. Note-se que este mix de métodos não se refere à diversidade dos métodos de coleta de dados. Já a operacionalização das variáveis referiu-se à descrição das variáveis consideradas no fenômeno estudado, além da sua forma 
de mensuração. Por fim, a dimensão de descrição dos procedimentos metodológicos teve como ponderação se o artigo apresentava os detalhes metodológicos aplicados na consecução da pesquisa.

A princípio a análise dos gráficos da Figura 4 revela que, de modo geral, nos artigos analisados, há um movimento ligeiramente crescente no refinamento do desenho de pesquisa. Contudo, ao ser examinado o comportamento evolutivo das facetas do desenho de pesquisa, fica saliente a baixa a utilização de mix de métodos de pesquisa, com especial queda nos últimos anos.

A variação positiva, no que se refere aos cuidados com o desenho de pesquisa, manifestou-se na presença mais marcante de operacionalização das variáveis de pesquisa. É clara a inversão das curvas de presença e ausência de operacionalização de variáveis de pesquisa, havendo predomínio da presença deste item a partir de 2001.

\section{Tipos de Dados Coletados}

Descrevem-se aqui os tipos de dados coletados e/ou utilizados pelas pesquisas descritas, ou seja, dados primários, coletados especificamente para a pesquisa; ou dados secundários, já existentes em outras fontes, coletados anteriormente. A Figura 5 demonstra a distribuição de frequiência dos artigos analisados por tipo de dado coletado/utilizado, considerando a possibilidade de aproveitamento de ambos os tipos no mesmo estudo.

Figura 5: Freqüência de Artigos por Tipo de Dado Coletado

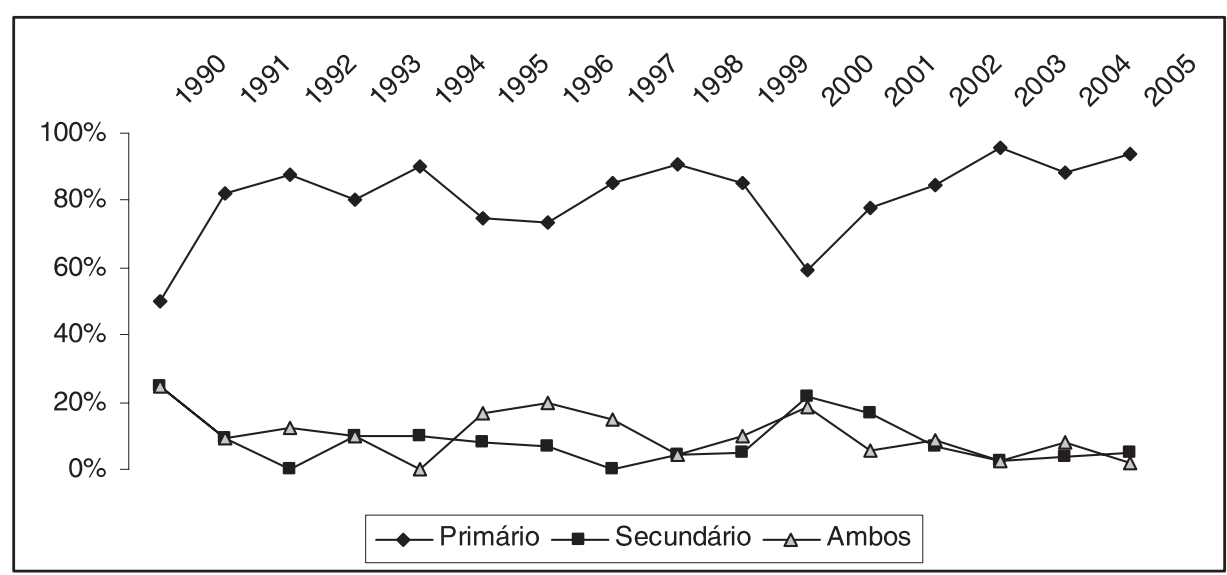

Nota: total de 407 artigos considerados como de base empírica e abordagem positivista.

Fonte: coleta de dados. 
Percebe-se claramente que a preferência dos pesquisadores tem sido e se tem firmado por dados primários, não se tendo alterado este perfil ao longo dos anos, afora a breve exceção de 2000 .

\section{Forma de Apresentação dos Resultados}

Relata-se aqui a forma como os resultados de pesquisa foram apresentados nos artigos analisados, isto é, se eles indicaram a validade externa e nomológica, bem como os limites do estudo e as recomendações aplicadas e para futuras pesquisas (Figura 6).

Quanto à validade externa, buscou-se avaliar em que medida os resultados obtidos pelos artigos poderiam aplicar-se a situações outras, além daquelas referentes ao estudo apresentado. Já no item relativo à validade nomológica, considerou-se a presença ou não de uma descrição da consistência dos resultados obtidos com o modelo teórico ou as hipóteses definidas a priori. Em relação aos limites do estudo e recomendações para pesquisas, verificou-se apenas a sua presença ou não nos artigos.

Na Figura 6, verifica-se que a preocupação dos autores com validade externa vem aumentando ao longo do tempo, dada a aproximação crescente entre a linha de ausência e a de presença de relato de validade externa nos artigos. Da mesma forma, no que se refere à apresentação nos artigos de discussão da validade nomológica, as curvas de presença e ausência se aproximavam, com tendência de crescimento maior na curva de registro de validade nomológica, até o ano de 2003, com leve afastamento posterior. Contudo cumpre registrar que os percentuais relativos a esses dois quesitos ainda são relativamente baixos.

Outra mudança clara se expõe na postura dos pesquisadores, ao projetarem os resultados de seus estudos aos seus públicos, nas recomendações da pesquisa. É notória a inversão de foco neste quesito, a partir do final da década de 90 , tanto no incremento positivo das suas recomendações para pesquisas futuras, quanto na redução proporcional das suas recomendações aplicadas ou implicações gerenciais.

Por fim, há sensível acréscimo, absoluto e percentual, no número de trabalhos com apresentação de limitações das pesquisas, configurando uma alteração clara no comportamento dos acadêmicos no período mais recente. 
Figura 6: Freqüência de Artigos por Tipo de Dado Coletado
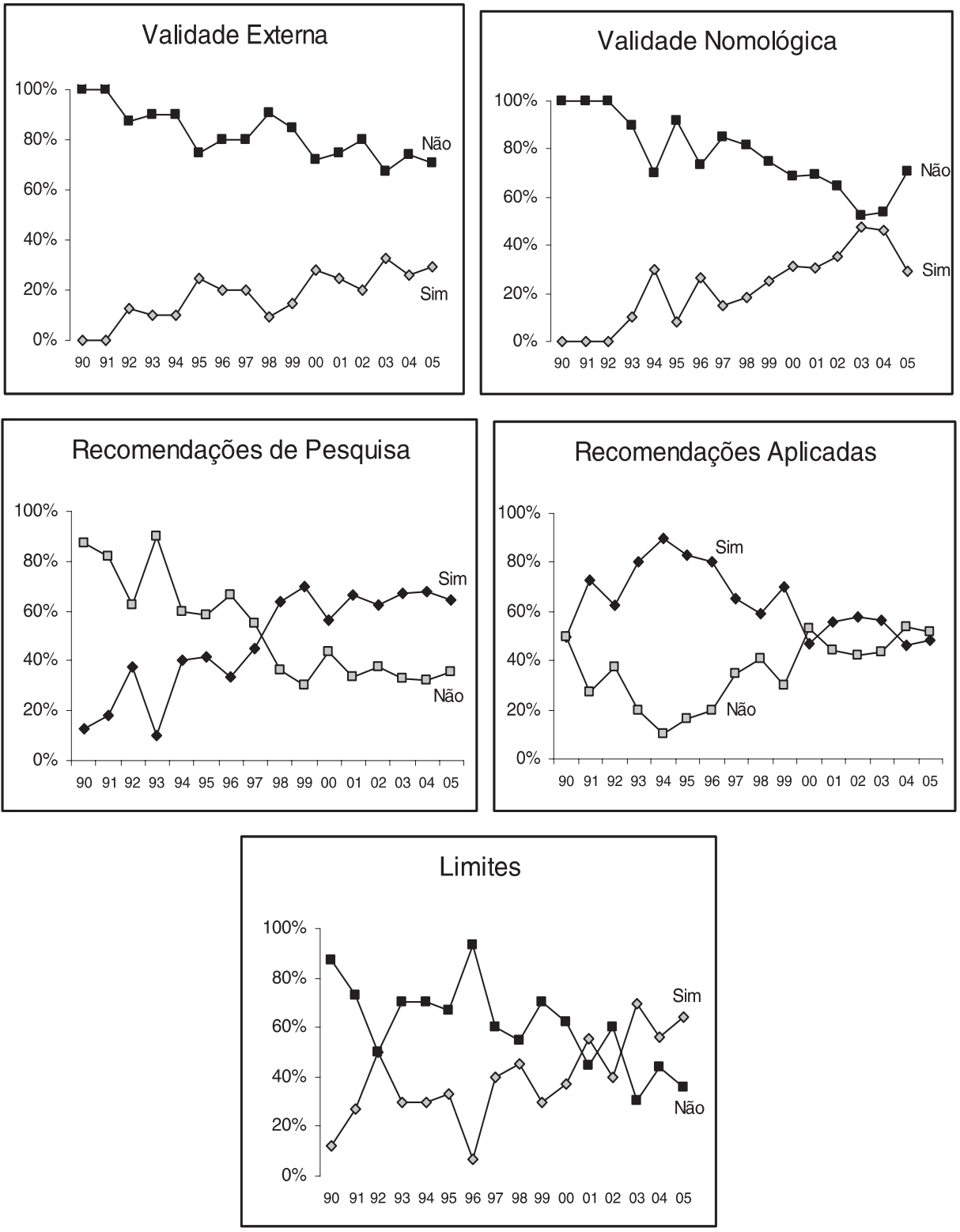

Nota: total de 407 artigos considerados como de base empírica e abordagem positivista.

Fonte: coleta de dados. 


\section{Síntese dos Resultados e a Comparação com o EMA}

Com o intuito de comparação, os resultados do levantamento foram sintetizados por período de tempo e comparados com o padrão de comportamento geral, identificado no primeiro congresso promovido pela ANPAD, especificamente para a área de Marketing no Brasil, EMA. O espaço de tempo de produção científica pesquisado (de 1990 a 2005) foi dividido em dois momentos, quais sejam, a década de 90 e os anos subseqüentes. Esta divisão se deu por dois motivos: a) uma intenção inicial de comparação dos resultados da pesquisa realizada por Perin et al. (2000); e b) a intenção de verificação de algum provável efeito sobre a produção científica do segundo momento, a partir dos primeiros artigos de posicionamento crítico sobre o statu quo da área de Marketing do Brasil no escopo dos ENANPAD (por exemplo, Casotti, 1998, 1999; Jaime Jr., 2000; Perin et al., 2000; Rocha et al., 1999; Rossi \& Silveira, 1999; Vieira, 1998, 1999, 2000).

Para tanto, no escopo dos ENANPADs, para os dois períodos de tempo estabelecidos, foram calculados de forma agregada os seus percentuais gerais, sintetizando cada uma das variáveis consideradas no estudo e abordadas ano a ano no item anterior. O mesmo procedimento foi realizado para o EMA, evidentemente para o seu único ano de realização (2004). Estes cálculos são apresentados nas Tabelas 1 e 2.

Tabela 1: Síntese dos Resultados

\begin{tabular}{r|cc|c}
\hline \multirow{2}{*}{ Tipo de Pesquisa } & \multicolumn{2}{|c|}{ ENANPADs } & \multirow{2}{*}{ EMA } \\
& $\mathbf{9 0 - 9 9}$ & $\mathbf{0 0 - 0 5}$ & \\
\hline Survey & $52,7 \%$ & $63,4 \%$ & $48,3 \%$ \\
Qualitativa & $11,7 \%$ & $13,7 \%$ & $18,3 \%$ \\
Experimental & $2,0 \%$ & $7,1 \%$ & $15,0 \%$ \\
Conceitual & $33,7 \%$ & $12,1 \%$ & $18,3 \%$ \\
Não Positivista & & $3,7 \%$ & \\
\hline
\end{tabular}

Nota: total de 527 artigos considerados.

Fonte: coleta de dados.

Em resumo, a Tabela 1 aponta predomínio geral das pesquisas do tipo survey, com ascensão das pesquisas experimentais, aparentemente substituindo o lugar dos artigos conceituais, além de relativa estabilidade das pesquisas qualitativas. 
Chama a atenção, ainda no âmbito dos ENANPADs, a presença relevante de estudos de abordagem não positivista. No que tange ao comportamento percebido no EMA, nota-se um relativo fôlego das pesquisas qualitativas e experimentais, bem como dos artigos conceituais, apresentados no EMA, apesar do predomínio ainda relevante das pesquisas do tipo survey.

Considerados apenas os artigos identificados como de cunho positivista e de base empírica (Tabela 2), percebe-se que o EMA demonstrou comportamento similar àquele diagnosticado no período 2000 a 2005 nos ENANPADs. Em termos gerais, registra-se: a) predomínio de pesquisas de natureza descritiva e crescimento do número de pesquisas experimentais; b) preocupação crescente com o aprofundamento e registro das teorias de base dos estudos; c) atenção constante quanto à apresentação dos objetivos dos estudos, bem como dos procedimentos metodológicos aplicados; d) relativa manutenção do volume de artigos com exposição de hipóteses ou pressupostos de base e no tipo de dados utilizados nas pesquisas; e) incremento na presença de modelos de pesquisa, ou seja, o posicionamento do objeto em estudo na base teórica; f) redução da utilização de mix de métodos e das recomendações aplicadas das pesquisas; e g) aumento razoável no registro da forma de operacionalização das variáveis dos estudos e nas considerações dos limites das pesquisas, bem como no apontamento de recomendações de pesquisa e na manifestação de verificação de validades externa e nomológica, apesar de ainda serem percentualmente baixos.

Algumas saliências, contudo, podem ser destacadas na comparação entre ENANPADs e EMA. No âmbito dos ENANPADs, percebe-se claramente o fortalecimento dos estudos de natureza causal, a manutenção da hegemonia descritiva e a queda da aplicação da natureza exploratória. Note-se que no EMA, pelo contrário, parte dos estudos descritivos é preterida pelos estudos qualitativos e mais fortemente pelos estudos causais, em comparação imediata com os números do período mais próximo dos ENANPADs (2000 a 2005). Assinale-se, ademais, aparente foco mais acadêmico do EMA, bem como preocupação de teste de teorias de base, supostamente envolvendo relações entre construtos, na medida em que se verifica maior índice de trabalhos de natureza causal, maior número de artigos com explicitação de hipóteses, maior constância de descrição da operacionalização de variáveis e também mais intensidade na recomendação para pesquisas futuras e menor percentual de recomendações aplicadas. 
Tabela 2: Síntese dos Resultados

\begin{tabular}{|c|c|c|c|c|}
\hline \multirow{2}{*}{\multicolumn{2}{|c|}{ DIMENSÃO }} & \multicolumn{2}{|c|}{ ENANPADs } & \multirow{2}{*}{ EMA } \\
\hline & & $90-99$ & $00-05$ & \\
\hline \multirow[t]{3}{*}{ Natureza da Pesquisa } & Exploratória & $39,0 \%$ & $16,2 \%$ & $22,4 \%$ \\
\hline & Descritiva & $52,2 \%$ & $59,8 \%$ & $44,9 \%$ \\
\hline & Causal & $8,8 \%$ & $24,0 \%$ & $32,7 \%$ \\
\hline \multirow[t]{2}{*}{ Justificativa } & Sim & $91,2 \%$ & $84,1 \%$ & $75,5 \%$ \\
\hline & Não & $8,8 \%$ & $15,9 \%$ & $24,5 \%$ \\
\hline \multirow[t]{3}{*}{ Teorias de Base } & Aprofundada & $40,4 \%$ & $59,8 \%$ & $57,1 \%$ \\
\hline & Superficial & $54,4 \%$ & $38,4 \%$ & $42,9 \%$ \\
\hline & Ausente & $5,1 \%$ & $1,8 \%$ & \\
\hline \multirow[t]{2}{*}{ Objetivos } & Sim & $98,5 \%$ & $91,9 \%$ & $91,8 \%$ \\
\hline & Não & $1,5 \%$ & $8,1 \%$ & $8,2 \%$ \\
\hline \multirow[t]{2}{*}{ Hipóteses } & Sim & $34,6 \%$ & $37,6 \%$ & $43,8 \%$ \\
\hline & Não & $65,4 \%$ & $62,4 \%$ & $56,3 \%$ \\
\hline \multirow{2}{*}{ Modelo de Pesquisa } & $\operatorname{Sim}$ & $15,4 \%$ & $25,1 \%$ & $20,4 \%$ \\
\hline & $N a \tilde{o}$ & $84,6 \%$ & $74,9 \%$ & $79,6 \%$ \\
\hline \multirow[t]{2}{*}{ Mix de Métodos } & Sim & $5,9 \%$ & $1,8 \%$ & $2,0 \%$ \\
\hline & Não & $94,1 \%$ & $98,2 \%$ & $98,0 \%$ \\
\hline \multirow[t]{2}{*}{ Operacional. das Variáveis } & Sim & $30,9 \%$ & $64,2 \%$ & $73,5 \%$ \\
\hline & Não & $69,1 \%$ & $35,8 \%$ & $26,5 \%$ \\
\hline \multirow[t]{2}{*}{ Descr. dos Procedimentos } & $\operatorname{Sim}$ & $91,2 \%$ & $95,6 \%$ & $100,0 \%$ \\
\hline & Não & $8,8 \%$ & $4,4 \%$ & \\
\hline \multirow[t]{3}{*}{ Tipo de Dados } & Primários & $81,6 \%$ & $85,2 \%$ & $77,1 \%$ \\
\hline & Secundários & $6,6 \%$ & $8,1 \%$ & $14,6 \%$ \\
\hline & Ambos & $11,8 \%$ & $6,6 \%$ & $8,3 \%$ \\
\hline \multirow[t]{2}{*}{ Validade Externa } & Sim & $13,2 \%$ & $26,9 \%$ & $16,3 \%$ \\
\hline & Não & $86,8 \%$ & $73,1 \%$ & $83,7 \%$ \\
\hline \multirow[t]{2}{*}{ Validade Nomológica } & Sim & $15,4 \%$ & $36,9 \%$ & $34,7 \%$ \\
\hline & Não & $84,6 \%$ & $63,1 \%$ & $65,3 \%$ \\
\hline \multirow[t]{2}{*}{ Limites } & Sim & $31,6 \%$ & $55,4 \%$ & $57,1 \%$ \\
\hline & Não & $68,4 \%$ & $44,6 \%$ & $42,9 \%$ \\
\hline \multirow[t]{2}{*}{ Recomendações de Pesquisa } & Sim & $42,6 \%$ & $64,6 \%$ & $73,5 \%$ \\
\hline & Não & $57,4 \%$ & $35,4 \%$ & $26,5 \%$ \\
\hline \multirow[t]{2}{*}{ Recomendações Aplicadas } & Sim & $70,6 \%$ & $51,7 \%$ & $32,7 \%$ \\
\hline & Não & $29,4 \%$ & $48,3 \%$ & $67,3 \%$ \\
\hline
\end{tabular}

Nota: total de 407 artigos considerados como de base empírica e abordagem positivista.

Fonte: coleta de dados. 


\section{Discussão dos Resultados e Conclusões do Estudo}

Os resultados aqui apresentados ratificam as conclusões oferecidas no conjunto de estudos de diagnóstico que vêm sendo realizados no Brasil, ao longo dos últimos anos. Como destaque maior, evidencia-se a hegemonia da abordagem positivista, com intensa carga de levantamentos do tipo survey, com ênfase descritiva.

Tal situação de predomínio da linha quantitativa positivista é sustentada por uma série de estudos e avaliações apresentadas no âmbito do próprio ENANPAD. Assim, Farias (2004, p. 3) afirma que o "paradigma positivista parece preponderar, e as metodologias de cunho quantitativo". Também Kovacs et al. (2004, p. 13) afirmam que "a grande maioria dos estudos empíricos publicados na área de Marketing do ENANPAD foi de ordem quantitativa", sustentando esta constatação no fato de a "orientação dominante na área de marketing estar fortemente alinhada à tradição positivista".

Uma possível explicação é oferecida por Cerchiaro, Sauerbronn e Ayrosa (2004, p. 1), para os quais "os pesquisadores da área se têm dedicado a conceptualizar seus trabalhos e apresentar seus resultados em bases estritamente positivistas, temendo certamente a reprovação por parte de seus pares e a prostração acadêmica". Vilas Boas, Sette e Abreu (2004, p. 7), referindo-se a Anderson (1983), destacam que "uma visão positivista dominante é colocada como barreira [...] à aceitação de teorias alternativas de estudo".

Razão alternativa para tal predomínio pode ser justificada pela constante replicação ou adaptação de modelos já desenvolvidos, especialmente tendo como foco a produção científica de Marketing norte-americana, eminentemente positivista. Essa posição é defendida por Faria (2004, p. 2), ao afirmar que "a produção acadêmica em marketing no Brasil ainda é majoritariamente subordinada ao que é produzido nos EUA". Nesse sentido, ao analisar o cenário de produção científica nacional na área de Marketing e a postura dos pesquisadores nacionais, Faria (2004, p. 4) questiona "como obter reputação para si e para sua instituição sem reproduzir ou citar trabalhos produzidos nos EUA por pesquisadores daquele país?"

Com o objetivo de "identificar o quanto esta visão americana influencia a construção do conhecimento de Marketing no Brasil", Vieira et al. (2002, p. 2) desenvolveram estudo cujo resultado apontou que "a produção nacional reproduz as oscilações e os modismos de inspiração quase que exclusivamente norteamericana" (p. 9). Segundo Farias (2004, p. 11), com relação à produção nacional, 
"o que ocorre na maioria das vezes são releituras de produções internacionais". A explicação para isto, conforme Farias (2004, p. 5), é a de que:

O primeiro passo para publicar no Brasil é ter um artigo inédito, bem escrito, com uma temática atual, utilizando muita referência bibliográfica internacional (atenção, está se falando da área de marketing), provavelmente replicar uma escala americana, um estudo realizado nos Estados Unidos, recentemente publicado no Journal of Marketing. Isto já ajuda muito na probabilidade de aceitação do artigo.

Contudo, apesar da orientação positivista, os resultados não mostram atendimento efetivo dos critérios de qualidade de uma pesquisa empírico-positivista, especialmente no que se refere à validade das pesquisas (vide percentuais na Tabela 2). Neste sentido, Kovacs et al. (2004, p. 13), a partir de avaliação dos artigos apresentados na área de Marketing dos ENANPADs no período de 1998 a 2002, constatam que "existe pouca preocupação dos pesquisadores em relação a procedimentos de validade e de confiabilidade" nos artigos avaliados e que "muito pouco se tem feito acerca de tais procedimentos, o que se poderia considerar inaceitável numa academia que fosse madura, sobretudo dentro da orientação positivista".

Para Cardoso e Faria (2004, p. 4), "o fato é que a disciplina vem sendo dominada pelo paradigma lógico-empiricista no qual são enfatizadas [...] características como racionalidade, objetividade, universalidade e mensurabilidade". Na opinião de Cerchiaro, Sauerbronn e Ayrosa (2004, p. 1), "muitos pesquisadores na área de marketing parecem preocupados com o uso de modelos estatísticos, mensurações e testes de validade (Froemming et al., 2000a, 2000b; Vieira 1998, 1999, 2000, 2003), que só levam a generalizações e não ao desenvolvimento de novas teorias, reproduzindo aqui modelos desenvolvidos em outros países". Ao analisar o processo de publicação do pesquisador nacional da área de Marketing, Farias (2004, p. 3) parece raciocinar no mesmo sentido, alertando para "o processo de elaboração de artigos em massa" e salientando que "o que importa é publicar em grande quantidade [...]", num processo de produção estilo "linha de montagem".

Uma solução tem sido constantemente apresentada para a situação supracitada: que a aplicação da pesquisa seja de ênfase qualitativa (Rossi \& Hor-Meyll, 2001; Vieira et. al, 2002). Contudo, apesar da ampla discussão sobre esta alternativa, aparentemente sua efetividade não tem sido notada (vide situação da pesquisa qualitativa na Figura 1). Para Kovacs et al. (2004, p. 13), "apesar do crescimento do campo qualitativo, sua participação relativa na área de Marketing do referido 
congresso não tem acompanhado tal tendência". Também Cardoso e Faria (2004, p. 1) compartilham esta noção, ao relatarem que "no Brasil, mais especificamente, não percebemos grande produção acadêmica em torno de abordagens alternativas, que se mostrem mais adequadas à nossa realidade".

Ao avaliarem a pesquisa qualitativa usual, na subárea específica do comportamento do consumidor, considerando os dados resultantes da aplicação das técnicas de grupos de foco, entrevistas em profundidade e técnicas projetivas, Rossi e Hor-Meyll (2001, p. 5) assinalam que o tratamento destes dados "leva a resultados pobres, já que não dizem o suficiente a respeito do significado do produto em relação à vida do consumidor". Na mesma linha, Campomar (2005, p. 4) enfaticamente afirma que "esta idéia de se colocar números em pesquisas qualitativas acaba deturpando todo o processo" e que "a pesquisa qualitativa requer um protocolo muito claro do que foi feito, de tal forma que se possa atingir aquilo que é chamado de método científico".

Por outro lado, Cerchiaro, Sauerbronn e Ayrosa (2004, p. 2) buscam explicação para "tal predominância de estudos de caráter positivista", ao afirmarem que isto "ocorre provavelmente porque os pesquisadores da área de marketing não recebem treinamento adequado para bem realizar pesquisas qualitativas ou de caráter interpretativo".

É preciso, no entanto, destacar a busca de alternativas, mais recentemente, pela abordagem da fenomenologia, seja por meio de artigos de discussão sobre o tema (por exemplo: Barros, 2002; Botelho \& Macera, 2001; Casotti, 1998; Cerchiaro, Sauerbronn, \& Ayrosa, 2004; Jaime Jr., 2000; Neves \& Giglio, 2004; Rocha \& Barros, 2004; Rocha, Barros, \& Pereira, 2005; Vilas Boas, Sette, \& Abreu, 2004), seja pela produção de pesquisas e artigos empíricos com esta abordagem. Os dados aqui analisados mostram crescimento linear do percentual de artigos que relatam estudos empíricos com base em abordagem fenomenológica, registrando algo como 5,3\% dos trabalhos do ENANPAD em 2005.

Partindo do pressuposto de que a produção nacional é fortemente influenciada pela produção norte-americana, conforme supracitado, seria bastante plausível argumentar que um movimento dos pesquisadores americanos no sentido da fenomenologia tivesse reflexos na produção nacional. Para Barros (2002, p. 13), "os anos 90 já mostraram significativo aumento do número de publicações [em journals americanos da área de Marketing] em relação aos anos 80, de 6 para 24, o que gera uma expectativa de que na atual década o interesse se intensifique ainda mais". 
O argumento básico para a mudança de comportamento é este: "a proliferação de bons estudos de natureza qualitativa, onde cultura e consumo ganham enfoque integrado, tende a minar essas resistências e fortalecer uma linha de investigação que privilegie tal abordagem" (D`Angelo, 2003, p. 11). Para Cardoso e Faria (2004, p. 11), o "principal propósito argumentativo é incentivar a constituição ou o fortalecimento de uma abordagem de investigação no Brasil que seja menos ingênua ou de viés social, política ou culturalmente", não se tratando, segundo os autores, "de um argumento que visa a desafiar completamente as linhas de pesquisa tradicionais".

Vilas Boas, Sette e Abreu (2004, p. 1) postulam que a interdisciplinaridade é "fundamental na evolução e na busca de soluções para as necessidades do marketing da atualidade", conquanto evidenciem "o crescimento da utilização de perspectivas de coleta e análise de dados com base em estruturas cognitivas, relacionadas portanto a um paradigma interpretativista".

Conforme Neves e Giglio (2004, p. 2), "the use of qualitative data is not new in marketing research [...] The novelty is the growing interest in ethnography, the traditional anthropological methodology [...] trying to understand and to uncover the wholeness of his or her existence". Nesta linha, Rocha, Barros e Pereira (2005, p. 7), ao sustentarem a aplicação da abordagem fenomenológica, afirmam que "o consumo é um fenômeno complexo, simbólico e cultural, que não pode ser reduzido a esquemas causais ou experiências de laboratório simplificadoras, de cunho positivista".

\section{Considerações Finais}

O foco maior deste artigo esteve na avaliação da evolução das características metodológicas básicas da produção científica da área de Marketing no Brasil. A sua principal conclusão foi a de que a análise dos artigos de base empírica publicados nos anais do I EMA e dos ENANPADs, no período de 1990 a 2005, em termos gerais, sinaliza uma busca de aprofundamento dos conhecimentos e da base conceitual da área de Marketing no Brasil.

Percebe-se uma alteração de padrões diagnosticados em estudos anteriores por Perin et al. (2000) e por Froemming et al. (2000a, 2000b). Mais especificamente, em relação aos métodos de pesquisa aplicados pelo corpo de pesquisadores, apesar da manutenção de predomínio das pesquisas do tipo survey, 
é visível o crescimento da aplicação da metodologia experimental, além do despertar para a aplicação de abordagem alternativa à positivista. Já quanto à natureza da pesquisa, percebe-se avanço da abordagem causal em detrimento do caráter exploratório da pesquisa, além da tradicional supremacia das pesquisas de âmbito descritivo. Também como decorrência do incremento de pesquisas causais e do uso de experimentos, além do domínio das pesquisas surveys de foco descritivo, observa-se a manifestação de aprofundamento teórico dos estudos e de maior cuidado em delimitar o objetivo e a questão de pesquisa, bem como maior atenção à operacionalização das variáveis de estudo. Por outro lado, nota-se uma dificuldade clara na confecção de pesquisas de cunho qualitativo, bem assim a aplicação de mais de um método de pesquisa.

Por fim, importa registrar a curiosa constância na desatenção à discussão da validade externa e nomológica dos estudos realizados, apesar de pequeno incremento percentual ao longo do tempo. Em sentido contrário, destaca-se o positivo avanço no desenvolvimento de maiores recomendações para futuras pesquisas acadêmicas.

\section{Limites do Estudo}

Apesar do rigor aplicado na realização da presente pesquisa, deve-se relacionar como limitações deste estudo os seguintes pontos:

- As conclusões do estudo estão restritas às publicações do EMA e dos ENANPADs e ao período considerado.

- A subjetividade implícita de cada avaliador em suas análises individuais pode ter representado conclusões distintas para uma mesma situação, apesar dos critérios claros de análise e das reuniões de revisão de dúvidas.

\section{Pesquisas Futuras}

A partir dos resultados deste estudo, sugerem-se as seguintes pesquisas futuras:

. Comparação dos resultados da área de marketing com outras áreas.

. Comparação dos resultados nacionais com resultados de outros países.

Avaliação específica e detalhada por tipo de método: survey, qualitativa, experimental e fenomenológica. 


\section{Referencias Bibliográficas}

Anderson, P. F. (1983, Fall).

Marketing, scientific progress and scientific method. Journal of marketing, 47(2), 18-31.

Barros, C. F. (2002).

Marketing e etnografia: um levantamento em journals dos anos 80 e 90. Anais do Encontro Nacional dos Programas de Pós-Graduação em Administração, Salvador, BA, 26.

Botelho, D., \&

Macera, A. (2001).

Análise metateórica de teses e dissertações da área de marketing apresentadas na EAESP-FGV (19741999). Anais do Encontro Nacional dos Programas de Pós-Graduação em Administração, Campinas, SP, 25.

Caldas, M. P.,

Tinoco, T., \&

Chu, R. A. (2003).

Análise bibliométrica dos artigos de RH publicados no EnANPAD, na década de 1990: um mapeamento a partir das citações dos heróis, endogenias e jactâncias que fizeram a história recente da produção científica da área. Anais do Encontro Nacional dos Programas de Pós-Graduação em Administração, Atibaia, SP, 27.

Campomar, M. C. (2005).

Pesquisa em marketing e seus problemas versus pesquisa de marketing. Anais do Encontro Nacional dos Programas de PósGraduação em Administração, Brasília, DF, 29.
Cardoso, M. L., \&

Faria, A. (2004).

Em busca da aproximação da disciplina à realidade nacional. Anais do Encontro de Marketing da ANPAD, Porto Alegre, RS, $1^{\circ}$.

Cardoso, R. L.,

Pereira, C. A., \&

Guerreiro, R. (2004).

A produção acadêmica em custos no âmbito do EnANPAD: uma análise de 1998 a 2003. Anais do Encontro Nacional dos Programas de PósGraduação em Administração, Curitiba, PR, 28.

Casotti, L. (1998).

Marketing moderno e consumidor pós-moderno. Anais do Encontro Nacional dos Programas de PósGraduação em Administração, Foz do Iguaçu, PR, 22.

Casotti, L. (1999).

O que é a pesquisa do consumidor? reflexões geradas a partir de um problema prático. Anais do Encontro Nacional dos Programas de PósGraduação em Administração, Foz do Iguaçu, PR, 23.

Cerchiaro, I.,

Sauerbronn, J., \&

Ayrosa, E. (2004).

Uma visão alternativa da pesquisa em marketing: como a fenomenologia pode contribuir para gerar conhecimento em marketing. Anais do Encontro de Marketing da ANPAD, Porto Alegre, RS, $1^{\circ}$. 
Churchill, G. A., Jr. (1999).

Marketing Research: methodological foundation ( 7 th ed.). Orlando-FL: The Dryden Press.

D’Angelo, A. (2003).

Cultura e consumo: apanhado teórico e reflexões para o ensino e a pesquisa de marketing e administração. Anais do Encontro Nacional dos Programas de Pós-Graduação em Administração, Atibaia, SP, 27.

Faria, A. (2004).

Em busca de relevância no âmbito de estratégia de marketing. Anais do Encontro Nacional dos Programas de Pós-Graduação em Administração, Curitiba, PR, 28.

Farias, S. A. (2004).

Em busca da inovação no marketing brasileiro: discutindo o processo de publicação de artigos em revistas e congressos. Anais do Encontro de Marketing da ANPAD, Porto Alegre, $\mathrm{RS}, 1^{\circ}$.

Froemming, L. M. S.,

Luce, F. B.,

Perin, M. G.,

Sampaio, C. H.,

Beber, S. J. N., \&

Trez G. (2000a, maio/agosto).

Inventário de artigos científicos na área de marketing. Revista de Administração Contemporânea, 4(2), 159-173.

Froemming, L. M. S.,

Luce, F. B.,

Perin, M. G.,

Sampaio, C.H.,

Beber, S. J. N., \&

Trez G. (2000b, setembro/dezembro).

Análise da qualidade dos artigos científicos da área de marketing no Brasil: as pesquisas survey na década de 90. Revista de Administração Contemporânea, 4(3), 201-219.

Gonçalves, N. G.,

Gonçalves, S. A., \&

Augusto, P. O. M. (2004).

Sobre a relação Estado e educação na produção acadêmica brasileira (19712000): temas, críticas e expectativas. Anais do Encontro Nacional dos Programas de Pós-Graduação em Administração, Curitiba, PR, 28.

Hoppen, N.,

Lapointe, L., \&

Moreau, E. (1997)

Avaliação de artigos de pesquisa em sistemas de informação: proposta de um guia. Anais do Encontro Nacional dos Programas de Pós-Graduação em Administração, Foz do Iguaçu, PR, 22.

lizuka, E. S., \&

Sano, H. (2004).

$\mathrm{O}$ terceiro setor e a produção acadêmica. Anais do Encontro Nacional dos Programas de PósGraduação em Administração, Curitiba, PR, 28.

Jaime, P., Jr. (2000).

Breves notas sobre etnomarketing, ou das relações entre a antropologia do consumo e a administração mercadológica. Anais do Encontro Nacional dos Programas de PósGraduação em Administração, Florianópolis, SC, 24. 
Kovacs, M. H.,

Leão, A. L. M. S. de,

Vieira, R. S. G.,

Barbosa, L., \&

Dias, C. M. de (2004).

Podemos confiar nos resultados de nossas pesquisas? Uma avaliação dos procedimentos metodológicos nos artigos de marketing do EnANPAD. Anais do Encontro de Marketing da ANPAD, Porto Alegre, RS, $1^{\circ}$.

Neves, L. M. P., \&

Giglio, E. A. M. (2004).

Ethnographic marketing: a critical appraisal of the use of anthropology in marketing research. Anais do Encontro Nacional dos Programas de Pós-Graduação em Administração, Curitiba, PR, 28.

Perin, M. G.,

Sampaio, C.H.,

Froemming, L. M. S., \&

Luce, F. B. (2000).

A pesquisa survey em artigos de marketing nos ENANPADs da década de 90. Anais do Encontro Nacional dos Programas de Pós-Graduação em Administração, Florianópolis, SC, 24.

Proença, E. R.de,

Lopes, F. T. P., \&

Meucci, A. (2005).

A construção do conhecimento em marketing: considerações sobre o discurso acadêmico e a produção científica do I Encontro de Markting da ANPAD. Anais do Encontro Nacional dos Programas de PósGraduação em Administração, Brasília, DF, 29.
Révillion, A. S. P. (2001).

A utilização de pesquisas exploratórias na área de marketing. Anais do Encontro Nacional dos Programas de Pós-Graduação em Administração, Campinas, SP, 25.

Rocha, E. P. Q.,

Blajberg, C.,

Ouchi, C.,

Ballvé, F.,

Soares J.,

Bellia, L., et al. (1999).

Cultura e consumo: um roteiro de estudos e pesquisas. Anais do Encontro Nacional dos Programas de Pós-Graduação em Administração, Foz do Iguaçu, PR, 23.

Rocha, E. P. Q., \&

Barros, C. (2004).

Dimensões culturais do marketing: teoria antropológica, estudos etnográficos e comportamento do consumidor. Anais do Encontro Nacional dos Programas de PósGraduação em Administração, Curitiba, PR, 28.

Rocha, E. P. Q.,

Barros, C., \&

Pereira, C. (2005).

Perspectivas do método etnográfico em marketing: consumo, comunicação e etnografia. Anais do Encontro Nacional dos Programas de PósGraduação em Administração, Brasília, DF, 29. 
Rossi, C.A. V., \&

Hor-Meyll, L. F. (2001).

Explorando novas trilhas na pesquisa do consumidor. Anais do Encontro Nacional dos Programas de PósGraduação em Administração, Campinas, SP, 25.

Rossi, C. A. V., \&

Silveira, T. (1999).

Pesquisa sobre culturas nacionais: o inexorável futuro do marketing na globalização. Anais do Encontro Nacional dos Programas de PósGraduação em Administração, Foz do Iguaçu, PR, 23.

Tonelli, M. J.,

Caldas, M. P.,

Lacombe, B. M. B., \&

Tinoco, T. (2004).

O mapa da partilha: análise das áreas de comportamento organizacional e gestão de pessoas antes e depois da cisão da área de recursos humanos no EnANPAD, 1991-2003. Anais do Encontro Nacional dos Programas de Pós-Graduação em Administração, Curitiba, PR, 28.

\section{Vieira, F. G. D. (1999).}

Ações empresariais e prioridades de pesquisa em marketing: tendências no Brasil e no mundo segundo a percepção dos acadêmicos brasileiros. Anais do Encontro Nacional dos Programas de PósGraduação em Administração, Foz do Iguaçu, PR, 23.
Vieira, F. G. D. (2003, janeiro/março).

Narciso sem espelho: a publicação brasileira de marketing. Revista de Administração de Empresas, 43(1), 81-90.

Vieira, F. G. D. (2000).

Panorama acadêmico científico e temáticas de estudos de marketing no Brasil. Anais do Encontro Nacional dos Programas de Pós-Graduação em Administração, Florianópolis, SC. 24.

Vieira, F. G. D. (1998).

Por quem os sinos dobram? Uma análise da publicação científica na área de marketing no ENANPAD. Anais do Encontro Nacional dos Programas de Pós-Graduação em Administração, Foz do Iguaçu, PR, 22.

Vieira, R.,

Dias, C.,

Filho, J., \&

Neto, M. (2002).

O conhecimento de marketing sob os olhos da teoria crítica. Anais do Encontro Nacional dos Programas de Pós-Graduação em Administração, Salvador, BA, 26.

Vilas Boas, L. H.,

Sette, R., \&

Abreu, N. (2004).

Teoria e pesquisa em marketing: a contribuição da antropologia para o estudo do comportamento do consumidor. Anais do Encontro Nacional dos Programas de PósGraduação em Administração, Curitiba, PR, 28. 


\section{Apêndice A - Formulário Aplicado}

\begin{tabular}{|c|c|c|c|}
\hline \multicolumn{4}{|l|}{ CLASSIFICAÇÃO } \\
\hline \multicolumn{4}{|l|}{ AUTOR (ES) : } \\
\hline \multicolumn{4}{|l|}{ TITTULO: } \\
\hline \multicolumn{4}{|l|}{ PERIOODICO : } \\
\hline TIPO & Tipo da pesquisa & \multicolumn{2}{|c|}{$\begin{array}{l}\text { ( ) Survey } \\
\text { ( ) Qualitativa } \\
\text { ( ) Experimental } \\
\text { ( ) Conceitual } \\
\text { ( ) Não positivista }\end{array}$} \\
\hline NATUREZA & Natureza da pesquisa & \multicolumn{2}{|c|}{$\begin{array}{l}\text { Exploratória } \\
\text { Descritiva } \\
\text { Causal }\end{array}$} \\
\hline \multirow{4}{*}{$\begin{array}{l}\text { QUESTÃO } \\
\text { DE PESQUISA }\end{array}$} & Justificativa & Sim & Não \\
\hline & Teorias de base & \multicolumn{2}{|c|}{$\begin{array}{l}\text { Aprofundada } \\
\text { Superficial } \\
\text { Ausente ou não relacionada }\end{array}$} \\
\hline & Objetivo / Questão de pesquisa & Sim & Não \\
\hline & Hipóteses / pressupostos de base & Sim & Não \\
\hline \multirow{4}{*}{$\begin{array}{l}\text { DESENHO } \\
\text { DE PESQUISA }\end{array}$} & Modelo de pesquisa & Sim & Não \\
\hline & Mix de métodos & Sim & Não \\
\hline & Operacionalização das variáveis & Sim & Não \\
\hline & $\begin{array}{l}\text { Descrição dos procedimentos } \\
\text { metodológicos }\end{array}$ & Sim & Não \\
\hline \multirow{6}{*}{$\begin{array}{l}\text { INSTRUMENTOS DE } \\
\text { MEDIDA E COLETA } \\
\text { DOS DADOS } \\
\text { APRESENTAÇÃO } \\
\text { DOS RESULTADOS }\end{array}$} & Tipo de dados & \multicolumn{2}{|c|}{$\begin{array}{l}\text { Primários } \\
\text { Secundários } \\
\text { Ambos }\end{array}$} \\
\hline & Validade externa & Sim & Não \\
\hline & Validade nomológica & Sim & Não \\
\hline & Limites do estudo & Sim & Não \\
\hline & Recomendações para pesquisas & Sim & Não \\
\hline & Recomendações aplicadas & Sim & Não \\
\hline
\end{tabular}

